\title{
Biologische Mechanismen antidepressiver Wirksamkeit von körperlicher Aktivität
}

\author{
Norbert-Ullrich Neumann und Karel Frasch \\ Bezirkskrankenhaus Günzburg, Abt. Psychiatrie II der Universität Ulm
}

psychoneuro 2005; 31 (10): 513-518

D epressive Erkrankungen weisen die höchste Prävalenz aller psychischen Störungen auf und liegen auch in der Häufigkeitsskala aller Erkrankungen an vorderer Stelle (3, 39). Bewährte pharmakotherapeutische und psychotherapeutische Interventionen stehen zur Verfügung, dennoch bleiben viele depressive Erkrankungen unzulänglich behandelt. Ein großes Problem besteht auch hinsichtlich der Compliance bei antidepressiver Medikation. Entsprechende Untersuchungen konnten zeigen, dass bis zu 60\% aller Patienten die begonnene antidepressive Medikation bereits drei Wochen nach Behandlungsbeginn wieder von sich aus beenden $(4,28)$.

Auf der Suche nach weiteren effektiven, tolerablen und für die Betroffenen akzeptablen Behandlungsmethoden wurde die antidepressive Wirkung körperlicher (sportlicher) Aktivitäten entdeckt (14, 20, 30, 36, $39,50)$. In den kontrollierten, randomisierten Studien wurde dabei vorwiegend aerobe Dauerleistung „running“ oder „walking“ untersucht. Die zeitliche Intensität der Übungen lag zwischen drei bis fünf Mal pro Woche und 20 bis 60 Minuten pro Trainingseinheit. Die Dauer der Therapiestudien schwankte zwischen sechs und 20 Wochen. Untersuchungen an Patienten mit bipolaren Störungen liegen nicht vor. Die diagnostische Operationalisierung und die Erfassung der Symptomausprägung erfolgte mit sehr unterschiedlichen Instrumenten. Diese reichen von DSM-IV

Untersuchungen an depressiven Patienten legen antidepressive Effekte aerober kontinuierlicher körperlicher Aktivität nahe (z.B. Jogging, Walken). Auf der anderen Seite ist bisher ungeklärt, über welche neurobiologischen Mechanismen eine solche Wirkung erzielt wird; humanmedizinische Untersuchungen zu dieser Fragestellung liegen nicht vor. Ergebnisse tierexperimenteller Studien weisen darauf hin, dass körperliche Aktivität u. a. mit einem signifikanten Anstieg zerebraler Monoamine und neurotropher Faktoren (Brain Derived Neurotrophic Factor BDNF) korreliert. Letzteres wiederum bewirkt verstärkte Neurogenese speziell im Hippocampus. In der Grundlagenforschung haben Neurogenese und Neuroplastizität in jüngster Zeit herausragende Bedeutung erlangt. Vielleicht eröffnet die Methode des Dauerleistungstrainings neue therapeutische Optionen nicht nur für depressive Störungen.

(Diagnostic and Statistcal Manual of Mental Disordes) über RDC (Research Diagnostic Criteria) bis zu BDI (Beck Depression Inventory) und CIS (Clinical Inteview Scale).

Über welche physiologischen, neurobiologischen Mechanismen neuromuskuläre Aktivität zu antidepressiven Effekten führt bzw. führen kann, ist noch nicht geklärt. Basierend auf den bekannten biologischen Depressionstheorien, werden in der vorliegenden Arbeit entsprechende Hypothesen erstellt.

\section{Untersuchungen}

Nahezu alle der zahlreichen Untersuchungen $(13,14,20,30,36,38$, $39,50)$ kommen zu positiven Ergebnissen. Viele Untersuchungen weisen, wie in den Arbeiten von Brosse et al. (14), Craft et al. (20) und Lawler et al. (36) ausführlich dargestellt, erhebliche methodische Mängel auf. Auch finden sich nur vier methodisch einwandfreie Studien mit diagnostisch klar definierten Patientenkollektiven $(13,30,38,50)$. Die Arbeiten enthalten in der Regel keine Diskussion plausibler, neuro- biologischer Hypothesen hinsichtlich der (vermuteten) antidepressiven Effektivität körperlichen Trainings. In der Übersichtsarbeit von Brosse et al. (14) wird der mögliche Einfluss neuromuskulärer Aktivität auf Neurotransmittersysteme, neuroendokrine Funktionen und Endorphine diskutiert.

Martinsen et al. (38) und Veale et al. (50) fanden in kontrollierten Untersuchungen mit Patientengruppen (Tab. 1) gemessen am BDI signifikante Verbesserungen in der Therapiegruppe gegenüber der Kontrollgruppe. Blumenthal et al. (13) sowie Greist et al. (30) verglichen Laufgruppen mit jeweils zwei Kontrollgruppen. Diese waren zum einen eine medikamentös behandelte Gruppe und eine Gruppe mit Lauftherapie und Medikation, bzw. eine Gruppe mit zeitlich begrenzter Psychotherapie (insgesamt zehn Sitzungen mit zeitlichem Limit pro Sitzung) und eine Psychotherapiegruppe (insgesamt zehn Sitzungen) ohne zeitliche Begrenzung pro Sitzung. Alle Gruppen verbesserten sich im Verlauf signifikant, signifikante Unterschiede 
Tab. 1 Kontrollierte, randomisierte Studien an depressiven Patienten mit operationalisierter Diagnose

\begin{tabular}{|c|c|c|c|c|}
\hline Autoren & Gesamtgruppe & $\begin{array}{l}\text { Untersuchungsgruppen } \\
\text { und Art der Intervention }\end{array}$ & $\begin{array}{l}\text { Dauer der } \\
\text { Studie }\end{array}$ & Ergebnis \\
\hline $\begin{array}{l}\text { Blumenthal } \\
\text { et al. }\end{array}$ & 156 & $\begin{array}{l}\text { 1. Lauftraining } \\
\text { 2. Medikation } \\
\text { 3. Lauftraining und Medikation }\end{array}$ & 16 Wo. & $\begin{array}{l}\text { Keine Unterschiede } \\
\text { zwischen den Gruppen }\end{array}$ \\
\hline $\begin{array}{l}\text { Greist } \\
\text { et al. }\end{array}$ & 49 & $\begin{array}{l}\text { 1. Lauftraining } \\
\text { 2. zeitlich (pro Sitzung) } \\
\text { begrenzte Psychotherapie } \\
\text { 3. zeitlich (pro Sitzung) } \\
\text { unbegrenzte Psychotherapie }\end{array}$ & 12 Wo. & $\begin{array}{l}\text { Keine Unterschiede } \\
\text { zwischen den Gruppen }\end{array}$ \\
\hline $\begin{array}{l}\text { Martinsen } \\
\text { et al. }\end{array}$ & 49 & $\begin{array}{l}\text { 1. Lauftraining } \\
\text { 2. Routineversorgung }\end{array}$ & 9 Wo. & $\begin{array}{l}\text { Therapiegruppe signifikant } \\
\text { besser als Kontrollgruppe }\end{array}$ \\
\hline $\begin{array}{l}\text { Veale } \\
\text { et al. }\end{array}$ & 83 & $\begin{array}{l}\text { 1. Lauftraining } \\
\text { 2. Routineversorgung }\end{array}$ & 12 Wo. & $\begin{array}{l}\text { Therapiegruppe signifikant } \\
\text { besser als Kontrollgruppe }\end{array}$ \\
\hline
\end{tabular}

zwischen den Gruppen fanden sich nicht.

Verbindliches fehlt bezüglich der anzuwendenden Methode, d.h. es bleibt offen, ob Dauerlauf, Walken, Schwimmen, Laufbandtraining, Tanzen, Turnen, Gymnastik, Krafttraining etc. das geeignete Mittel ist (meist wurde aerobes Dauerleistungstraining - Laufen - untersucht). In welcher zeitlichen Einzelintensität, wie oft pro Tag oder Woche und für wie lange insgesamt zu trainieren ist, um die besten antidepressive Effekte zu erzielen, ist ebenfalls ungeklärt. Für das antidepressive Mittel „körperliche Aktivität“ gibt es also noch keine indikativen „Präparate“ und keine „Dosierungen“.

Abgesehen von diesen Unklarheiten bestehen auch noch immer grundsätzliche Zweifel hinsichtlich der Effektivität. Die meisten Untersuchungen erfüllen nicht die Kriterien, die an methodisch einwandfreie, replizierbare Untersuchungen zu stellen sind. Es fehlen operationalisierte Diagnostik, valide und reliable Psychopathometrie, Kontrollgruppen, Cross-
over-Design und Follow-up-Untersuchungen. Lawlor et al. (36), die in ihrer Übersicht nach gründlicher Vorauswahl 17 „randomisierte, kontrollierte Untersuchungen“ einer kritischen Analyse unterzogen haben, kommen zu dem Schluss, dass „die meisten Studien von minderer Qualität sind, kein Follow-up-Design haben und an einer mangelhaft operationalisierten, nicht klinischen Population vorgenommen wurden". Sie schreiben weiter, „körperliches Training mag bei der depressiven Symptomreduktion kurzfristig wirksam sein, die Wirksamkeit an einer klinischen Population bleibt ungeklärt". Zu ähnlich kritischen Beurteilungen kommen Brosse et al. (14) und Craft et al. (20) in ihren Übersichten. Brosse et al. stellen fest, dass in den vergangenen zehn Jahren lediglich drei randomisierte klinische Studien zu diesem Thema publiziert wurden, und nur eine dieser Untersuchungen eine ausreichend große Fallzahl sowie Nachuntersuchungen vorweisen kann.

Kurzfristige, mit der Trainingsdauer korrelierende, antidepressive

\section{Tab. 2 Pathophysiologische und pathoanatomische Grundlagen der Depression}

- Neurotransmitter- und Neurorezeptor-Hypothesen

- Neuroendokrine Hypothesen

Hypothalamus-Hypophysen-Nebennierenrindenachse

Hypothalamus-Hypophysen-Schilddrüsenachse

- Durchblutungs- und Stoffwechselanomalien

- Strukturelle Veränderungen

Neurogenese/Neuroplastizität

Effekte werden aber auch von den Kritikern für wahrscheinlich gehalten. Auf die zugrunde liegenden biologischen Wirkmechanismen solcher Effekte gehen aber auch sie nicht oder nur kurz und unvollständig ein.

Orientiert man sich an den verschiedenen Theorien zur Pathophysiologie der Depression (Tab. 2), eröffnen sich, insbesondere basierend auf Ergebnissen tierexperimenteller Untersuchungen, verschiedene Erklärungsmodelle für die antidepressiven Effekte körperlicher Aktivität.

\section{Neurobiologische Mechanismen neuro- muskulärer Aktivität}

Bisher kann keine Neurotransmitter- und Rezeptorstörung für sich genommen, keine hormonelle Störung, kein pathologisch-anatomischer Befund und kein Befund der funktionellen Bildgebung allein die Pathophysiologie der Depression mit ihren affektiven, kognitiven und vegetativ-somatischen Symptomen erklären.

Andererseits gibt es genügend klare Hinweise dafür, dass das limbische System - Amygdala-Hippocampuskomplex, Gyrus Cinguli - der präfrontrale Kortex, der Thalamus und hypothalamische Neuronenensembles sowie die direkte und indirekte monoaminerge Neurotransmission in diesen Arealen der Pathophysiologie der Depression zugrunde liegen.

Sowohl aus der humanmedizinischen Forschung wie insbesondere aus tierexperimentellen Untersu- 
chungen liegen Ergebnisse vor, welche die antidepressiven Effekte körperlicher Aktivität verständlich machen können:

- Erhöhung zerebraler Monoamine verbunden mit positiven Verhaltenseffekten

- Erhöhung von BDNF (Brain Derived Neurotrophic Factor) und vermehrte Neurogenese speziell im Hippocampus.

\section{Neurotransmitter- und Rezeptor- störungen}

Stand der neurobiologischen Forschung ist, dass neurochemischen Störungen der Reizübertragung im ZNS eine ausschlaggebende Rolle bei der Ätiopathogenese depressiver Erkrankungen zukommt. Die Hypothese einer verminderten serotonergen und/oder noradrenergen Neurotransmission wird durch die Pharmakodynamik der Antidepressiva gestützt. Auch die Neurotransmitter Dopamin, Acetylcholin und GABA sowie Signaltransduktionssysteme scheinen von Bedeutung zu sein (43).

Die Hypothese, dass körperliche Aktivität Synthese und Stoffwechsel der zerebralen Monoamine beeinflusst, unterstützen tierexperimentelle wie auch humane Untersuchungen. Letztere liefern allerdings nur Daten über Plasmaspiegelerhöhungen der Monoamine und entsprechender Metaboliten (16, 17, 37, 50). Rückschlüsse auf ZNS-Konzentrationen von Neurotransmittern sind anhand dieser Befunde nur bedingt möglich.

In einer Untersuchung wird über erhöhte periphere freie Fettsäuren und erhöhtes freies Tryptophan als Effekt körperlichen Trainings berichtet (11). Die Autoren spekulieren, dass eine vermehrte Verfügbarkeit der Aminosäurenvorläufer im ZNS die Syntheserate für Serotonin erhöhen könnte.

In tierexperimentellen Untersuchungen konnte mittels verschiedener Bewegungsmethoden (Laufrad, Stufenklettern, Schwimmen) eine signifikante Erhöhung zerebraler Monoamine ausgelöst werden, verbunden mit positiven Verhaltenseffekten $(22,25)$. Bemerkenswerterweise führte Stufenklet- tern zu einer selektiven Vermehrung von präfrontalem Noradrenalin, während Schwimmen in einer erhöhten serotonergen Neurotransmission resultierte.

Diese Befunde müssen insofern kritisch betrachtet werden als die Versuchstiere unter „erzwungenen“ Bedingungen Leistung erbracht haben, sozusagen in Stresssituationen. Die Ergebnisse könnten also weniger durch die körperliche Aktivität als vielmehr durch die Stressexposition erklärt sein (21).

\section{- Neuroendokrine Störungen}

Alterationen hormoneller Achsen finden sich bei etwa der Hälfte aller depressiven Patienten. Veränderungen in den hypothalamisch gesteuerten Regelkreisen, die die Ausschüttung der Kortikoide (HPAAchse), der Schilddrüsenhormone (HPT-Achse) und des Wachstumshormons kontrollieren, sind vielfach nachgewiesen (47).

Nachdem der HPA- und HPT-Regelkreis an der Pathophysiologie der Depression beteiligt sind, könnten Interventionen, die auf diesen Mechanismus zielen, antidepressive Effekte haben (34). Körperliches Training kann in der Tat die Stressanfälligkeit des HPA-Regelkreises stabilisieren. (12,52). Da depressive $\mathrm{Zu}-$ stände häufig von einer HPA-Hyperaktivität gekennzeichnet sind, könnte eine entsprechende HPA-Regelkreis-Normalisierung auch antidepressive Effekte beinhalten. Dieser mögliche antidepressive Effekt kann aber nur für einen Teil depressiver Patienten von Bedeutung sein, da nur in etwa der Hälfte der Fälle die erwähnte Hyperaktivität der HPA-Achse vorliegt (1).

Eine Verringerung der HPA-Überaktivität - sowohl der depressionsimmanenten wie der stressinduzierten - ist nicht nur aus Sicht der hormonellen Pathophysiologie von Bedeutung, sondern auch mit Blick auf hirnmorphologische Konsequenzen.

Während eine vorübergehende erhöhte Glukokortikoidausschüttung im Rahmen einer Stressreaktion physiologisch sinnvoll ist, führt eine anhaltende Hypersekretion zu hirnmorphologischen Veränderungen, vor allem im limbisch-hippocampa- len Bereich $(10,33)$. Insbesondere nimmt die Synthese von BDNF (brain derived neurotrophic factor) unter vermehrter Glukokortikoidausschüttung signifikant ab $(10,15,29)$. Die Atrophie funktionstragender hippocampaler Nervenzellen und die damit einhergehende Dysfunktion mit Blick auf die physiologische Fähigkeit des Hippocampus, die HPAAchse zu hemmen, führt schließlich zu einem Circulus vitiosus von anhaltend erhöhten Glukokortikoidspiegeln und hippocampalem Nervenzelluntergang. Intensives körperliches Training kann über erhöhte BDNF-Synthese $(18,19,27)$ diesem pathologischen Kreislauf entgegenwirken, was mittelbar in antidepressiver Effektivität resultieren könnte.

Eine latente oder manifeste $\mathrm{Hy}$ pothyreose wird als depressionsdisponierender Faktor erachtet (6). Schilddrüsenhormonsubstitution gilt als probates Mittel bei der Behandlung therapierefraktärer Depressionen $(2,5,7)$. Über modulierende Einflüsse von Dauerleistungstraining oder körperlicher Aktivität allgemein auf den HPT-Regelkreis oder die Schilddrüsenfunktion liegen keine Ergebnisse vor.

\section{Durchblutungs- und Stoff- wechselanomalien}

Funktionelle bildgebende Verfahren wie PET und SPECT, die die Bestimmung der regionalen Hirndurchblutung ermöglichen, zeigen bei Depressionen eine Reduktion der Hirndurchblutung und des Hirnstoffwechsels in anterioren Regionen, insbesondere im linken dorsolateralen präfrontalen Kortex. Des weiteren fielen Veränderungen von Durchblutung und Stoffwechsel im Bereich des anterioren und posterioren Cingulums und des medialen präfrontalen Kortex auf $(40,45)$.

Globale oder regionale zerebrale Perfusionsstörungen spielen für die aktuellen pathophysiologischen Depressionsmodelle im Gegensatz zum Glukosemetabolismus keine nennenswerte Rolle $(9,23)$. Zu letzterem liegen widersprüchliche Ergebnisse vor, wobei je nachdem ein frontaler oder ein cingulärer Hypo- oder Hypermetabolismus als depressionstypisch postuliert wird $(24,26,35)$. 
Intensive körperliche Aktivität führt u.a. zu signifikanter zerebraler Durchblutungssteigerung von regional unterschiedlicher Intensität (32). Vermehrte Durchblutung führt unmittelbar zu einem intensiveren Angebot aller nervenzellphysiologisch und -anatomisch notwendigen Substanzen und hat damit einen neuroprotektiven und -reparativen Effekt. Mittelbar könnte sich dies in einer Normalisierung verschiedenster depressiogener neuropathologischer und neuropathophysiologischer Gegebenheiten manifestieren.

\section{Strukturelle Veränderungen}

Eine Poststroke Depression korreliert eher mit anterioren und linkshirnigen Läsionen (31). Potenziell vulnerable Regionen scheinen der Amygdala-Hippocampuskomplex, der Gyrus cinguli, der präfrontale Kortex und die verbindenden Faszikel zu sein (46).

\section{Neurogenese (Zytoarchitektur/ Neuroplastizität)}

Tierexperimentelle Befunde lassen darauf schließen, dass ein Mindestmaß an neuromuskulärer Aktivität notwendig ist, um physiologische Werte von BDNF (Brain Derived Neurotrophic Factor) und damit das Potenzial der Neuroplastizität zu sichern $(29,42,44)$. Es konnte gezeigt werden, dass bei Ratten unter Laufbandbelastung schon nach kurzer Zeit der neurotrophe Faktor (BDNF) speziell im Hippocampus hochsignifikant zunahm (18, 19, 42). BDNFmRNA und BDNF-Proteine fanden sich aber auch vermehrt im Gyrus dentatus, im Hilus und in der CA3Region. Vergleichbares wurde auch in cerebellären und kortikalen Regionen festgestellt (10).

Adulte Neurogenese findet auch im menschlichen Gehirn statt (27). Zusammen mit der Neubildung von Dendriten und Synapsen begründet dies das enorme neuroplastische Potenzial. Der Umstand, dass körperliche Aktivität höchstwahrscheinlich auch beim Menschen die BDNFGenexpression in besonderem Maße fördert, wäre für die Depressionsbehandlung - und nicht nur für diese von großer Wichtigkeit. Sowohl das neuroprotektive wie das neurogene- tische BDNF-Potenzial speziell im hippocampalen Bereich spielen eine erhebliche Rolle mit Blick auf verschiedene Dimensionen der Pathophysiologie der Depression. Körperliches Training könnte also mittelbar über BDNF-Stimulation zur Normalisierung der HPA-Aktivität bei depressiven Zuständen beitragen und damit einem möglichen depressiogenen Pathomechanismus entgegenwirken. Der Umstand, dass es auch unter antidepressiver Medikation zu einer deutlichen Zunahme hippocampaler BDNF-mRNA-Konzentrationen kommt, stützt die Vermutung, dass darin ein (noch nicht weiter geklärter) antidepressiver Mechanismus gründet (48).

Der Hippocampus ist von herausragender Bedeutung für kognitive Prozesse, insbesondere für die Organisation des deklarativen Gedächtnisses. Dyskognitive Symptome sind in vielen Fällen integraler Bestandteil depressiver Erkrankungen (8). Zumindest die Besserung solcher depressiver Teilsymptome wäre über die wiederholt erwähnte trainingsinduzierte BDNF-Aktivierung und die damit einhergehende adulte hippocampale Neurogenese unmittelbar zu erklären $(27,49)$.

\section{- Fazit}

Hinsichtlich des geeigneten („antidepressiven“) Typs körperlicher Aktivität sprechen klinische Untersuchungen $(18,19,32,33)$ und tierexperimentelle Untersuchungen $(29,42,44,49)$ dafür, dass positive neurotrope Effekte nicht über statische oder anaerobe Leistungen (38) zu erzielen sind, sondern durch aerobe dynamische Dauerleistungen. Hollmann et al. (33) beschreiben die „allgemeine aerobe dynamische Ausdauer“ als eine „dynamische Beanspruchung unter Einsatz von mehr als mindestens $1 / 6$ der gesamten Skelettmuskulatur über eine Zeitspanne von mindestens fünf Minuten mit einer Belastungsintensität unterhalb von $70 \%$ der individuellen Höchstleistungsfähigkeit“.

Die Vermutung, dass körperliche Aktivität im Allgemeinen und gezieltes sportliches Dauerleistungstraining im Besonderen nicht nur gut für die körperliche Verfassung sind, sondern auch Geist und Seele beflügeln, reicht bis in die Antike ( "mens sana in corpore sano“). Die klinische Psychiatrie der jüngeren Vergangenheit und Gegenwart mit ihrer Zentrierung auf Psychotherapie und Pharmakotherapie sieht Spiel und Sport eher als allgemeine euthyme Beschäftigungsmaßnahme und misst ihr bisher keine spezifisch-therapeutische Effektivität zu. Die ersten Untersuchungen zum Thema Depressivität und körperliche Aktivität kamen auch nicht aus der psychiatrischen Forschungsrichtung, sondern seitens der Sportphysiologie (39). Dies hat sich in jüngster Zeit geändert. Zu zuverlässigen und schlüssigen Ergebnissen ist man dennoch noch nicht gekommen. In Sachen körperlicher Aktivität und neurobiologische Effekte kamen in den letzten Jahren bemerkenswerte Hinweise aus der experimentellen Neurophysiologie. Vor allem die Untersuchungen, die für einen engen Zusammenhang zwischen körperlicher Aktivität, Neurogenese und Neuroplastizität sprechen, liefern vielversprechende Ausblicke auch für die humane Neurowissenschaft.

Die präventive, therapeutische und rehabilitative Bedeutung von Sport und Bewegung für z.B. HerzKreislauf-Erkrankungen, Übergewicht, Bluthochdruck und Diabetes mellitus ist längst unbestritten. Mit Blick auf Gehirngesundheit und damit auch psychische Gesundheit öffnet sich nunmehr eine neue Dimension, deren Bedeutung noch nicht abzuschätzen ist.

\section{Biological antidepressive modes of action of physical exercise} Investigations in depressive patients suggested antidepressive effects of continuous aerobic physical exercise (e.g. jogging, walking). On the other hand, it is yet unclear which mode(s) of neurobiological action are responsible for this since there are no human studies addressing this question. Results of animal studies showed an increase of cerebral monoamines and the Brain Derived Neurotrophic Factor BDNF, which is associated with neuroneogenesis especially in the hippocampus. In today's basic research it has been repeatedly stated 
that neuroneogenesis and neuroplasticity are of outstanding relevance so that maybe continuous neuromuscular activity opens new therapeutic options not only for depressive disorders.

\section{Key words}

physical exercise - depression - cerebral monoamines - neurogenesis

\section{Literatur}

1. Akil $\mathrm{H}$ et al. Multiple HPA profiles in endogenous depression: effect of age and sex on cortisol and beta-endorpin. Biol Psychiatry $1993 ; 33: 73-85$

2. Altshule LL et al. Does thyroid supplementation accelerate tricyclic antidepressant response? A review and meta-analysis of the literature. Am J Psychiatry 2001; 158 : 1617-1622

3. Angst ]. Epidemiologie affektiver Störungen. Journal of the ZNS 1992; 1: 2-8

4. Aspler $\mathrm{R}$ et al. Correlates of compliance with psychoactive prescriptions. J Psychoactive Drugs 1984; 16: 193-199

5. Bauer M et al. Hochdosierte Thyroxinbehandlung bei therapie- und prophylaxeresistenten Patienten mit affektiven Psychosen. Nervenarzt 1998; 69: 1019-22

6. Bauer $\mathrm{M}$ et al. Thyroid hormone, neural tissue and mood modulation. World J Biol Psychiatry 2001; 2: 59-69

7. Bauer $M$ et al. Supraphysiological doses of L-thyroxine in the maintenance treatment of prophylaxis-resistant affective disorders. Neuropsychopharmacolpgy 2002; 27: 620 628

8. Beblo T et al. Neuropsychologische Defizite bei depressiven Störungen. Fortschr Neurol Psychiat 2000; 68: 1-11

9. Bench Cl et al. Regional cerebral blood flow in depression measured by positron emission tomography: the relationship with clinical dimensions. Psychol Med 1993; 23 : 579-590

10. Berchtold NC et al. Estrogen and exercise interact to regulate brain-derives neurotrophic factor mRNA and protein expression in the hippocampus. Eur J Neurosci 2001; 14: 1992-2002

11. Blomstrand $\mathrm{E}$ et al. Changes in plasma concentrations of aromatic and branchedchain amino acids during sustained exercise in man and their role in fatigue. Acta Physiol Scand 1988; 133: 115-121

12. Blumenthal JA et al. Stress activity and exercise training in premenopausal and postmenopausal women. Health Psychol 1991; 10: 384-391

13. Blumenthal JA et al. Comparison of aerobic exercise in older patients with major depression. Arch Intern Med 1999; 159 (19): 2349-2356

14. Brosse AL et al. Exercise and the treatment of clinical depression in adults. Sports Med 2002; 32: 741-760

15. Carro $E$ et al. Circulating insulin-like growth factor I mediates effects of exercise on the brain. J Neurosci 2000; 20: 2926-33

16. Chaouloff $F$. Effects of acute physical exercise on central serotonergic systems. Med Sci Sports Exerc 1997; 29: 58-62

17. Chaouloff F. Physical exercise and brain monoamines: a review. Acta Physiol Scand 1989; 137: 1-13

18. Cotman CW. Exercise: A behavioral intervention to enhance brain health and plasticity. Trends Neurosci 2002; 25: 295-301

19. Cotman CW et al. Exercise enhances and protects brain function. Exerc Sport Sci Rev 2002; 30: 75-79

20. Craft LL et al. The effect of exercise on clinical depression and depression resulting from mental illness: a metaanalysis. J Sport Exerc Psych 1998; 20: 339-357

21. Dishman RK. Brain monoamines, exercise and behavioral stress: animal models. Med Sci Sports Exerc 1997; 29: 63-74

22. Dishman RK et al. Treadmill exercise training augments brain norepinephrine response to familiar and novel stress. Brain Res Bull 2000; 52: 337-342

23. Drevets WC et al. A functional anatomical study of unipolar depression. J Neurosci 1992; 12: 3628-3641

24. Drevetz WC et al. Neuroanatomical circuits in depression: implications for treatment mechanisms. Psychopharmacol Bull 1992; 28: 261-274

25. Dunn AL et al. Brain norepinephrine and metabolites after treadmill training and wheel running in rats .Med Sci Sports Exerc 1996; 28: 204-209

26. Ebert $D$ et al. Role of the cingulate gyrus in depression: From functional anatomy to depression. Biol Psychiatry 1996; 39: $1044-$ 1050

27. Eriksson PS et al. Neurogenesis in the adult human hippocampus. Nat Med 1998; 4: $1313-7$

28. Fawcett J. Compliance: Definitions and key issues. J Clin Psychiatry 1995; 56: 4-8

29. Gomez-Pinilla F et al. Physical exercise induces FGF- 2 and its mRNA in the hippocampus. Brain Res 1997; 764: 1-8

30. Greist JH et al. Running as a treatment for depression. Compr Psychiatry 1979; 20 (1): 41-54

31. Herrmann M et al. Poststroke depression: Is there a pathoanatomic correlate for depression in the postacute stage of stroke? Stroke 1995; 26: 850-856

32. Hollmann W et al. Körperliche Aktivität fördert Gehirngesundheit und -leistungsfähigkeit - Übersicht und eigene Befunde. Nervenheilkunde 2003; 9: 467-474

33. Hollmann $\mathrm{W}$ et al. Zur Biochemie des Gehirns bei muskulärer Arbeit. Nervenheilkunde 1998; 17: 30-35

34. Holsboer $F$. The rationale for corticotropinreleasing hormone receptor (CRH-R) antagonists to treat depression and anxiety. J Psychiatr Res 1999; 33: 181-214

35. Ketter TA et al. Functional brain imaging, limbic function, and affective disorders. The Neuroscientist 1996; 2: 55-65

36. Lawlor DA et al.The effectiveness of exercise as an intervention in the management of depression: Systematic review and meta-regression analysis of randomised trials. BM] 2001; 322: 1-8

37. Lechin $\mathrm{F}$ et al. Plasma neurotransmitters, blood pressure, and heart rate during supine resting, orthostasis, and moderate exercise in dysthymic depressed patients. Biol Psychiatry 1995; 37: 884-891

38. Martinsen EW et al. Comparing aerobic with nonaerobic forms of exercise in the treatment of clinical depression: A randomized trial. Compr Psychiatry 1989; 30: $324-$ 331

39. Martinsen EW. Benefits of exercise for the treatment of depression. Sports Med 1990; 9: 380-389

40. Mayberg $\mathrm{HS}$ et al. Cingulate function in depression: a potential predictor of treatment response. Neuroreport 1997; 8: 1057-1061

41. Murray CJL et al. Global mortality, disability, and the contribution of risk factors: Global burden of disease study. Lancet 1997; 349: 1436-1442

42. Neeper SA et al. Physical activity increases mRNA for brain-derived neurotrophic factor and nerve growth factor in rat brain. Brain Res 1996; 726: 49-56

43. Nutt DJ. The neuropharmacology of serotonin and noradrenalin in depression. Int Clin Psychopharmacol 2002; 17 (Suppl 1): $1-12$

44. Russo-Neustadt AA et al. Physical activity and antidepressant treatment potentiate the expression of specific brain-derived neurotrophic factor transcripts in the rat hippocampus. Neuroscience 2000; 101: 305-312

45. Salloway $S$ et al. Subcortical disease and neuropsychiatric illness. J Neuropsychiatry Clin Neurosci 1994; 6: 93-99

46. Stern R et al. Depressive symptoms following stroke. Am J Psychiatry 1991; 154: 351-356

47. Ströhle A. Die Neuroendokrinologie von Stress und die Pathophysiologie und Therapie von Depression und Angst. Nervenarzt 2003; 74: 279-292

48. Swaab DF et al. Interaction of prefrontal cortical and hypothalamic systems in pathogenesis of depression. Prog Brain Res 2000; 126: 369-396

49. Tong $L$ et al. Effects of exercise on geneexpression profile in the rat hippocampus. Neurobiol Dis 2001; 8: 1046-56

50 . Veale $D$ et al. Aerobic exercise in the adjunctive treatment of depression: a randomised controlled trial. J R Soc Med 1992; 85 (9): 541-544

51. Weicker $\mathrm{H}$ et al. Influence of exercise on serotonergic neuromodulation in the brain. Amino Acids 2001; 20: 35-47

52. Wittert GA et al. Adaption of the hypothalamopituitary adrenal axis to chronic exercise stress in humans. Med Sci Sports Exerc 1996; 28: 1015-1019

\section{Korrespondenzadresse:}

PD Dr. med. N.-U. Neumann

Abt. Allgemeine Psychiatrie und

Psychotherapie des BKH Günzburg

an der Donau-Ries-Klinik

Neudegger Allee 6

86609 Donauwörth

Norbert.Neumann@bkh-guenzburg.de 